\title{
Factors Associated with Overweight and Obesity among Children of Mexican Descent: Results of a Binational Study
}

\author{
Lisa G. Rosas • Sylvia Guendelman • Kim Harley • \\ Lia C. H. Fernald · Lynnette Neufeld • \\ Fabiola Mejia $\cdot$ Brenda Eskenazi
}

Published online: 9 March 2010

(C) The Author(s) 2010. This article is published with open access at Springerlink.com

\begin{abstract}
The prevalence of childhood obesity is high among young children of Mexican origin in the United States, however, the determinants are poorly understood. We conducted a binational study with a sample from California (CA) and Mexico (MX), to identify and compare the most important factors associated with overweight and obesity among children of Mexican descent. Significantly more children were classified as overweight or obese in CA compared to MX (53.3 vs. $14.9 \%, P<0.01)$. In CA and MX, having an obese mother was significantly associated with being overweight or obese. In MX, male gender, high socioeconomic status and very low food insecurity were associated with being overweight or obese. These data offer hypotheses for how migration may influence the high prevalence of overweight among the Mexican children in California.
\end{abstract}

L. G. Rosas

Department of Family and Community Medicine, University of California, San Francisco, 3333 California St. Suite 365, San Francisco, CA 94118, USA

e-mail: rosasl@fcm.ucsf.edu

S. Guendelman · L. C. H. Fernald

Department of Community Health and Human Development, University of California, Berkeley, Berkeley, CA, USA

K. Harley · B. Eskenazi $(\bowtie)$

Center for Children's Environmental Health Research, University of California, Berkeley, 1995 University Ave. Suite 265, Berkeley, CA 94720-7392, USA

e-mail: eskenazi@berkeley.edu

L. Neufeld · F. Mejia

Department of Nutritional Epidemiology, Instituto Nacional de Salud Publica, Cuernavaca, Mexico
Keywords Children - Obesity · Mexican immigrants . Transnational

\section{Introduction}

The prevalence of childhood obesity has increased dramatically in the United States (US) over the past 30 years [1-4], especially among young children of Mexican origin [5]. Approximately 33\% of young Mexican-American children in the US are considered overweight or obese, which is higher than both non-Hispanic white $(25 \%)$ and black (24\%) children [6]. Overweight children are more likely to become overweight adults [7-9] and adults of Mexican origin are at increased risk for morbidities associated with obesity, such as diabetes, cardiovascular disease and uncontrolled hypertension compared to other racial and ethnic groups [10-14]. Yet the determinants of childhood obesity in this population are not well understood.

Acculturation is a frequently studied determinant of obesity among children of Mexican descent [15-27]. However, while the literature has supported an increased risk of obesity with increasing acculturation among Mexican-American adults [28-33], the association in children remains unclear [34]. A more complete understanding of the obesity epidemic among children of Mexican descent may come from transnational research (US-Mexico) given the close proximity of the two countries as well as the strong ties between immigrants and their communities of origin. The determinants of childhood obesity among children of Mexican descent are likely to be different depending on whether they reside in Mexico or the US [35]. Programs and policies aimed at preventing and reducing obesity among children of Mexican descent in the 
US will benefit from a better understanding of the factors that contribute to obesity on both sides of the border. Such a binational strategy was recommended by the Institute of Medicine in a recent report on preventing overweight in Latino children [36].

Mexico is currently undergoing a transition, where problems of nutritional deficit (stunting, micronutrient deficiencies) and infectious disease now coexist with an industrialized country profile of obesity and chronic disease $[35,37,38]$. As part of this transition, the prevalence of childhood overweight has been increasing [39] and studies in Northern Mexico and Mexico City have shown prevalence estimates similar to the US [40-42].

Using a transnational study design, the aim of this study was to identify and compare the most important factors associated with overweight and obesity among Mexicoborn and California-born children of Mexican descent.

\section{Methods}

\section{Study Design and Participants}

We conducted a binational study using two cross-sectional samples of 5-year-old children and their mothers in California and Mexico. The mothers and children from California were participants of the Center for the Health Assessment of Mothers and Children of Salinas (CHAMACOS) study, a longitudinal birth cohort in the agricultural Salinas Valley. Pregnant women were recruited from 1999 to 2000 in prenatal clinics serving a predominantly low-income, Spanish-speaking population. Detailed methods for this longitudinal study have been published previously [43, 44]. Of 601 women initially enrolled, 526 were followed through delivery of a live singleton birth that survived the neonatal period, and 350 follow-up interviews were completed when the children were 5-years-old. Mothers born in the US $(n=45)$ and children with incomplete anthropometric information $(n=18)$ were excluded for a final sample size of 287 mother-child pairs of whom all children were born in the US and all mothers born in Mexico. Children with missing anthropometric information were more likely to have obese mothers and mothers who were recent immigrants to the US than children without missing data $(P<0.05)$.

Mexican 5-year-old children were participants in the Proyecto Mariposa study. Proyecto Mariposa was designed to capture a sample in Mexico that closely resembled the California sample but had not migrated. In the California cohort, Mexican-born women were most commonly from the states of Guanajuato (20\%), Jalisco (12\%) and Michoacán $(24 \%)$ with other states of origin each representing less than $5 \%$ of the study population. Thus, Proyecto
Mariposa included women, who had never migrated themselves, and their 5-year-old children from highmigration municipalities in these states defined as having at least $10 \%$ of the male population residing in the US in the majority of towns and districts within the municipality as of the most recent Mexican census in 2005 [45]. We recruited women and their 5-year-old children who were beneficiaries of the social welfare program, Oportunidades, to obtain a population similar to the California sample that was also receiving government benefits (Special Supplemental Nutrition Program for Women, Infants and Children) and accessing health care. Of 317 mother-child pairs in the Proyecto Mariposa sample, 316 children had complete anthropometric information and were included in this analysis.

Institutional Review Boards at UC Berkeley and the National Public Health Institute in Mexico approved this study.

\section{Measures}

Face-to-face interviews were conducted and identical anthropometric protocols were followed in California and Mexico. Mothers provided informed consent for themselves and their children.

Outcome definition: Children were weighed and measured using calibrated electronic scales (Tanita MotherBaby Scale Model 1582, Tanita Corp.) and stadiometers. Each child was measured in triplicate and the average of the measurements was used. Standardization procedures were conducted in each site. In MX, interviewers participated in a standardization procedure before the study began at a local school where interviewers each measured the same child until agreement was reached. In CA, where interviewers had considerable experience conducting anthropometric assessments as a result of previous study visits at 6 months, 1, 2 and 3.5 years, we conducted pilot testing where the two different interviewers took measurements until agreement was reached. We calculated body mass index (BMI) as mass in kilograms divided by height in meters squared and for both samples, compared to sex-specific BMI-for-age percentile data issued by the Centers for Disease Control and Prevention (CDC) in 2000 [46]. We classified children who were at or above the 85th percentile but less than the 95th percentile as overweight and those at or above the 95th percentile as obese [46].

Independent variables: We calculated median daily hours playing outside and watching television based on mother's report of average time spent in these activities on a typical weekend and weekday. The questions regarding television viewing were part of the Home Observation for the Measurement of the Environment Short Form (HOME-SF), a previously validated and widely used questionnaire. 
Frequency of intake of foods shown to be associated with child weight in the literature [47] including sodas, other sweetened beverages, fast food, sweets and snacks, fruits and vegetables were assessed through maternal report using a quantitative food frequency questionnaire (FFQ). For food groups such as sweets and snacks as well as fruits and vegetables, the FFQ included the most commonly consumed items in the respective country.

Mother's height and weight were measured using an electronic Tanita scale (Tanita Mother-Baby Scale Model 1582, Tanita Corp.) and stadiometer. Mother's weight status was defined as overweight if her BMI was greater than or equal to 25 and less than 30 and obese if her BMI was 30 or greater [48]. Total time spent in the US was used as a proxy for acculturation in the California sample given that all women were foreign-born and the majority was monolingual Spanish-speaking [49]. In Mexico, women were asked if the child's father or the current head of household or any other close family member, including her grandparents, parents, siblings or other children had migrated to the US for work and if any of them were currently residing in the US.

Household food insecurity during the past 12 months was measured using the US Household Food Security Instrument, Spanish Version (Short Form) in both locations [50, 51]. Households were classified as: food secure, low food secure, and very low food secure according to US Department of Agriculture guidelines [50]. A different measure of socioeconomic status (SES) was used in each country that differentiated between low, medium and high SES levels within each sample, without reference to the larger community. Among the California mothers, 97\% were receiving WIC and the Mexico mothers were recruited form Oportunidades, which targets the lowest 20th percentile of economic well-being in the country. In the California study, SES was measured using a continuous measure of per capita household income divided into tertiles. In Mexico, a developing country where income measures do not accurately characterize SES [52], we used a principal component analysis to summarize housing characteristics and assets and the first principal component weightings were retained [53]; a weighted score was calculated for each women, and the weighted scores were divided into tertiles. Housing characteristics and assets included type of house (owned, rented, borrowed, shared, mortgaged, given as benefit of employment), floor (dirt, cement, wood/tile/other finish), sanitary service (none, latrine, toilet with no plumbing, toilet with plumbing), and kitchen (no separate room, a separate room where people also sleep, a separate room where no one sleeps), as well as televisions (none, black and white, color, more than one color), cars (none, one old, one new, more than one), number of household appliances (VCR, DVD player, CD player, tape player, microwave, washer, computer, printer, air conditioner and home phone) and number of light bulbs.

\section{Statistical Analysis}

Statistical analyses were conducted using STATA 10.0 for Windows [54].

We used chi-square tests and Fisher's exact tests in the case of small cell sizes to compare the California and Mexico samples according to several demographic and health behavior characteristics. Within the California sample, we also compared children according to mother's length of residence in the US.

We used logistic regression to determine and compare the most important determinants for being classified as overweight or obese ( $\geq 85$ th percentile) in California and Mexico. For the crude analysis, we chose demographic and behavioral variables that have been shown in the literature to be associated with children's weight status [47] or that we hypothesized a priori to be associated with children's weight status in this population. For the California sample, we repeated the crude analysis using the 95th percentile cutoff for obesity because of the high number of children in this category. For the multivariate logistic regression analysis, we considered variables for inclusion if they were related to the outcome $(P<0.1)$ in the crude analysis for either sample or if they were of interest a priori. For each sample, we used a manual backwards stepwise elimination procedure removing the variables from the model one at a time using the likelihood ratio test to determine if the model's fit improved using a cutoff of $P<0.05$. For the California sample, we reran the final model using the 95 th percentile cutoff.

\section{Results}

Compared to California-born children, Mexico-born children were more likely to have been breastfed exclusively for 6 months, to have a mother with fewer years of education and not actively engaged in the workforce, and to live in a household that experienced more food insecurity (Table 1). Compared to the Mexico-born children, children in California watched more television, ate fast food more frequently, and consumed more fruit, as reported by the mothers. Mothers in Mexico reported that their children consumed more soda and spent more time playing outside than mothers in California. Intake of sweets, snacks and vegetables did not differ between groups.

The prevalence of overweight and obesity in mothers was high in both samples, with obesity significantly higher in California (49\%) compared to Mexico (33\%) $(P<0.01)$. More than $40 \%$ of mothers in Mexico reported 
Table 1 Selected socio-demographic characteristics and health behaviors of participants, California (Salinas, CA) and Mexico (Guanajuato, Jalisco, and Michoacán, Mexico) 2006

\begin{tabular}{|c|c|c|c|c|c|c|c|c|c|c|c|c|}
\hline \multirow[t]{3}{*}{ Characteristic/behavior } & \multirow{2}{*}{\multicolumn{2}{|c|}{$\begin{array}{l}\text { Mexico } \\
n=316\end{array}$}} & \multirow{2}{*}{\multicolumn{2}{|c|}{$\begin{array}{l}\text { California } \\
n=287\end{array}$}} & \multirow[t]{3}{*}{$P$-value } & \multicolumn{6}{|c|}{ California: mother's years in the US } & \multirow[t]{3}{*}{$P$-value } \\
\hline & & & & & & \multicolumn{2}{|c|}{$\begin{array}{l}5-10 \text { years } \\
n=160\end{array}$} & \multicolumn{2}{|c|}{$\begin{array}{l}11-15 \text { years } \\
n=92\end{array}$} & \multicolumn{2}{|c|}{$\begin{array}{l}16 \text { years or more } \\
n=53\end{array}$} & \\
\hline & $n$ & $(\%)$ & $n$ & $(\%)$ & & $n$ & $(\%)$ & $n$ & $(\%)$ & $n$ & $(\%)$ & \\
\hline Child sex & & & & & 0.98 & & & & & & & 0.80 \\
\hline Male & 149 & $(47.2)$ & 135 & $(47.0)$ & & 71 & $(49.0)$ & 40 & $(44.9)$ & 24 & $(45.3)$ & \\
\hline Female & 167 & $(52.9)$ & 152 & $(53.0)$ & & 74 & $(51.0)$ & 49 & $(55.1)$ & 29 & $(54.7)$ & \\
\hline Exclusively breastfed $\geq 6$ months & & & & & $<0.01$ & & & & & & & 0.47 \\
\hline No & 216 & $(68.4)$ & 250 & $(87.7)$ & & 128 & $(88.9)$ & 75 & $(84.3)$ & 47 & $(90.4)$ & \\
\hline Yes & 100 & $(31.7)$ & 35 & $(12.3)$ & & 16 & $(11.1)$ & 14 & $(15.7)$ & 5 & (9.6) & \\
\hline Mother's weight status & & & & & $<0.01$ & & & & & & & $0.10^{\mathrm{b}}$ \\
\hline Normal & 78 & $(24.7)$ & 37 & $(12.9)$ & & 24 & $(16.6)$ & 9 & $(10.1)$ & 4 & (7.6) & \\
\hline Overweight & 132 & $(41.8)$ & 110 & $(38.3)$ & & 61 & $(42.1)$ & 32 & $(36.0)$ & 17 & $(32.1)$ & \\
\hline Obese & 106 & $(33.5)$ & 140 & $(48.8)$ & & 60 & $(41.4)$ & 48 & $(53.9)$ & 32 & $(60.4)$ & \\
\hline Mother's education & & & & & $<0.01$ & & & & & & & 0.30 \\
\hline Elementary or less & 217 & $(68.7)$ & 142 & $(49.5)$ & & 66 & $(45.5)$ & 51 & $(57.3)$ & 25 & $(47.2)$ & \\
\hline Middle or high school & 93 & $(29.4)$ & 101 & $(35.2)$ & & 52 & $(35.9)$ & 27 & $(30.3)$ & 22 & $(41.5)$ & \\
\hline High school graduate or more & 6 & (1.9) & 44 & $(15.3)$ & & 27 & $(18.6)$ & 11 & $(12.4)$ & 6 & $(11.3)$ & \\
\hline Married or living as married & & & & & 0.05 & & & & & & & 0.66 \\
\hline No & 21 & (6.7) & 32 & $(11.2)$ & & 14 & $(9.7)$ & 12 & $(13.5)$ & 6 & $(11.3)$ & \\
\hline Yes & 295 & $(93.4)$ & 255 & $(88.9)$ & & 131 & $(90.3)$ & 77 & $(86.5)$ & 47 & $(88.7)$ & \\
\hline Mother's work status & & & & & $<0.01$ & & & & & & & $0.27^{\mathrm{b}}$ \\
\hline Not working & 203 & $(64.0)$ & 86 & $(28.2)$ & & 48 & $(30.0)$ & 27 & $(29.4)$ & 11 & $(20.8)$ & \\
\hline Work less than $20 \mathrm{~h} /$ week & 54 & $(17.0)$ & 15 & $(4.9)$ & & 6 & $(3.8)$ & 4 & $(4.4)$ & 5 & (9.4) & \\
\hline Work $20-40 \mathrm{~h} /$ week & 22 & $(6.9)$ & 68 & $(22.3)$ & & 31 & $(19.4)$ & 20 & $(21.7)$ & 17 & $(32.1)$ & \\
\hline Work more than $40 \mathrm{~h} /$ week & 38 & $(12.0)$ & 136 & $(44.6)$ & & 75 & $(46.9)$ & 41 & $(44.6)$ & 20 & $(37.7)$ & \\
\hline Household food insecurity & & & & & $<0.01$ & & & & & & & $0.50^{\mathrm{b}}$ \\
\hline Secure & 78 & $(24.7)$ & 172 & $(59.9)$ & & 93 & $(64.1)$ & 50 & $(56.2)$ & 29 & $(54.7)$ & \\
\hline Low food security & 149 & $(47.2)$ & 87 & $(30.3)$ & & 39 & $(26.9)$ & 28 & $(31.5)$ & 20 & $(37.7)$ & \\
\hline Very low food security & 89 & $(28.2)$ & 28 & $(9.8)$ & & 13 & $(9.0)$ & 11 & $(12.4)$ & 4 & (7.6) & \\
\hline \multicolumn{13}{|l|}{ Family member currently in US ${ }^{a}$} \\
\hline No & 176 & $(55.7)$ & & & & & & & & & & \\
\hline Yes & 140 & $(44.3)$ & & & & & & & & & & \\
\hline Daily TV time & & & & & $<0.01$ & & & & & & & 0.36 \\
\hline $1 \mathrm{~h}$ or less & 138 & $(43.7)$ & 85 & $(29.6)$ & & 49 & $(33.8)$ & 26 & $(29.2)$ & 10 & $(18.9)$ & \\
\hline $1-2 \mathrm{~h}$ & 85 & $(26.9)$ & 101 & $(35.2)$ & & 47 & $(32.4)$ & 33 & $(37.1)$ & 21 & $(39.6)$ & \\
\hline Greater than $2 \mathrm{~h}$ & 93 & $(29.4)$ & 101 & $(35.2)$ & & 49 & $(33.8)$ & 30 & $(33.7)$ & 22 & $(41.5)$ & \\
\hline Time spent playing outside & & & & & $<0.01$ & & & & & & & 0.78 \\
\hline $1 \mathrm{~h}$ or less & 41 & $(13.0)$ & 113 & $(39.4)$ & & 57 & $(39.3)$ & 34 & $(38.2)$ & 22 & $(41.5)$ & \\
\hline $2-3 \mathrm{~h}$ & 141 & $(44.6)$ & 124 & $(43.2)$ & & 59 & $(40.7)$ & 41 & $(46.1)$ & 24 & $(45.3)$ & \\
\hline $4 \mathrm{~h}$ or more & 134 & $(42.4)$ & 50 & $(17.4)$ & & 29 & $(20.0)$ & 14 & $(15.7)$ & 7 & $(13.2)$ & \\
\hline Soda consumption $^{\mathrm{c}}$ & & & & & $<0.01$ & & & & & & & $0.20^{\mathrm{b}}$ \\
\hline Less than 1 per week & 73 & $(23.1)$ & 110 & $(38.3)$ & & 59 & $(40.7)$ & 27 & $(30.3)$ & 24 & $(45.3)$ & \\
\hline $1-6$ per week & 189 & $(59.8)$ & 153 & $(53.3)$ & & 77 & $(53.1)$ & 53 & $(59.6)$ & 23 & $(43.4)$ & \\
\hline 1 a day or more & 54 & $(17.1)$ & 24 & $(8.4)$ & & 9 & $(6.2)$ & 9 & $(10.1)$ & 6 & $(11.3)$ & \\
\hline Other sweetened beverage consumption ${ }^{\mathrm{c}}$ & & & & & $<0.01$ & & & & & & & 0.37 \\
\hline Less than 1 per day & 196 & $(62.0)$ & 112 & $(39.0)$ & & 64 & $(44.1)$ & 29 & $(32.6)$ & 19 & $(35.9)$ & \\
\hline 1 to less than 2 per day & 71 & $(22.5)$ & 54 & $(18.8)$ & & 26 & $(17.9)$ & 16 & $(18.0)$ & 12 & $(22.6)$ & \\
\hline 2 or more perday & 49 & $(15.5)$ & 121 & $(42.2)$ & & 55 & $(37.9)$ & 44 & $(49.4)$ & 22 & $(41.5)$ & \\
\hline
\end{tabular}


Table 1 continued

\begin{tabular}{|c|c|c|c|c|c|c|c|c|c|c|c|c|}
\hline \multirow[t]{3}{*}{ Characteristic/behavior } & \multirow{2}{*}{\multicolumn{2}{|c|}{$\begin{array}{l}\text { Mexico } \\
n=316\end{array}$}} & \multirow{2}{*}{\multicolumn{2}{|c|}{$\begin{array}{l}\text { California } \\
n=287\end{array}$}} & \multirow[t]{3}{*}{$P$-value } & \multicolumn{6}{|c|}{ California: mother's years in the US } & \multirow[t]{3}{*}{$P$-value } \\
\hline & & & & & & \multicolumn{2}{|c|}{$\begin{array}{l}5-10 \text { years } \\
n=160\end{array}$} & \multicolumn{2}{|c|}{$\begin{array}{l}11-15 \text { years } \\
n=92\end{array}$} & \multicolumn{2}{|c|}{$\begin{array}{l}16 \text { years or more } \\
n=53\end{array}$} & \\
\hline & $n$ & $(\%)$ & $n$ & $(\%)$ & & $n$ & $(\%)$ & $n$ & $(\%)$ & $n$ & $(\%)$ & \\
\hline Fast food consumption & & & & & $<0.01$ & & & & & & & 0.07 \\
\hline Less than once per week & 271 & $(86.0)$ & 104 & $(36.5)$ & & 60 & $(42.0)$ & 31 & $(34.8)$ & 13 & $(24.5)$ & \\
\hline Once a week or more & 44 & $(14.0)$ & 181 & $(63.5)$ & & 83 & $(58.0)$ & 58 & $(65.2)$ & 40 & $(75.5)$ & \\
\hline Sweets and fried snacks consumption ${ }^{c}$ & & & & & 0.31 & & & & & & & 0.08 \\
\hline Less than 1 per day & 58 & $(18.4)$ & 41 & $(14.3)$ & & 16 & $(11.0)$ & 16 & $(18.0)$ & 9 & $(17.0)$ & \\
\hline 1 to less than 2 per day & 93 & $(29.4)$ & 81 & $(28.2)$ & & 50 & $(34.5)$ & 16 & $(18.0)$ & 15 & $(28.3)$ & \\
\hline 2 or more perday & 165 & $(52.2)$ & 165 & $(57.5)$ & & 79 & $(54.5)$ & 57 & $(64.0)$ & 29 & $(54.7)$ & \\
\hline Fruit consumption $^{\mathrm{c}}$ & & & & & $<0.01$ & & & & & & & $0.39^{\mathrm{b}}$ \\
\hline Less than 1 per day & 113 & $(35.8)$ & 16 & (5.6) & & 5 & (3.5) & 7 & (7.9) & 4 & (7.6) & \\
\hline $1-2$ per day & 115 & $(36.4)$ & 43 & $(15.0)$ & & 24 & $(16.6)$ & 10 & $(11.2)$ & 9 & $(17.0)$ & \\
\hline 2 per day or more & 88 & $(27.9)$ & 228 & (79.4) & & 116 & $(80.0)$ & 72 & $(80.9)$ & 40 & $(75.5)$ & \\
\hline Vegetable consumption $^{\mathrm{c}}$ & & & & & 0.25 & & & & & & & 0.47 \\
\hline Less than 1 per day & 25 & (7.9) & 34 & (11.9) & & 13 & $(9.0)$ & 15 & $(16.9)$ & 6 & $(11.3)$ & \\
\hline $1-2$ per day & 82 & $(26.0)$ & 68 & $(23.7)$ & & 35 & $(24.1)$ & 19 & $(21.4)$ & 14 & $(26.4)$ & \\
\hline 2 per day or more & 209 & $(66.1)$ & 185 & $(64.5)$ & & 97 & $(66.9)$ & 55 & $(61.8)$ & 33 & $(62.3)$ & \\
\hline
\end{tabular}

that their partner or other close family member was currently living in the US with California as the most common destination state reported (data not shown). The length of time mothers in California had lived in the US at the time of the interview was not significantly associated with any of the demographic characteristics, physical activity indicators, or dietary intake levels.

Significantly more children were classified above the 85th percentile in California compared to Mexico (53.3 vs. $14.9 \%, P<0.01$ ) (Fig. 1). No significant differences in weight status were detected in the California sample by mother's years in the US.

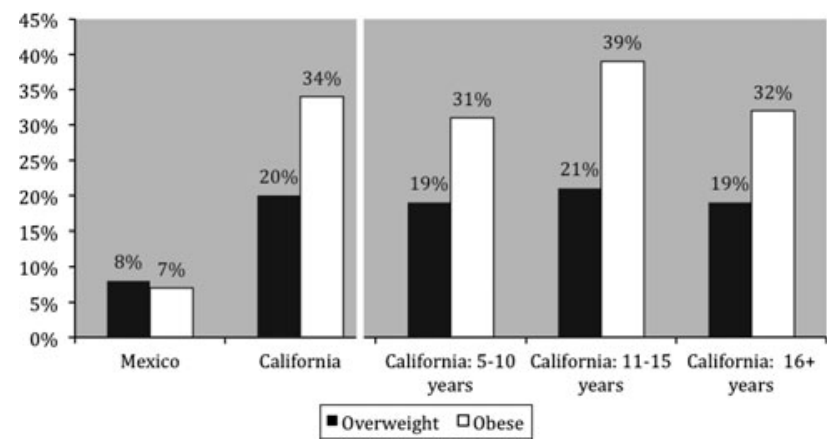

Fig. 1 Children's weight status in Mexico, California, and California by mother's years in US
As shown in Tables 2 and 3, in the California sample, having an obese mother was significantly associated with being overweight or obese ( $>85$ th percentile of BMI) in both the crude (OR $2.595 \%$ CI 1.2, 5.2) and adjusted analysis (OR $2.495 \%$ CI 1.1, 5.3). Although there was a non-significant trend $(P=0.1)$ towards higher prevalence of overweight among children of mothers who had been in the US longer (11-15 years vs. 5-10 years), this association disappeared in the multivariate model. When we reran the California model separately using the 95th percentile cutoff instead of the 85 th percentile (data not shown), our findings for the crude and adjusted analysis did not change significantly.

Among the Mexico-born children, having an obese mother also significantly increased the odds of the child being overweight or obese in both the crude (OR $5.495 \%$ CI: 1.8, 16.3) and adjusted models (OR 5.5 95\% CI: 1.8, 17.1). Very few children, however, who were classified above the 85 th percentile had mothers in the normal weight category $(n=4)$ so these analyses should be interpreted with caution. In both the crude and adjusted analyses for the Mexico sample, we found that children's weight status was significantly associated with sex, socioeconomic status, and household food insecurity. In the multivariate model, girls had half the odds of being classified above the 85th percentile compared to boys (OR $0.595 \%$ CI: 0.2 , $0.9)$; children in the highest SES tertile had three times the 
Table 2 Selected characteristics and behaviors and crude odds ratios with 95\% confidence intervals for weight status, California (Salinas, CA) and Mexico (Guanajuato, Jalisco, and Michoacán, Mexico) 2006

\begin{tabular}{|c|c|c|c|c|c|c|c|c|c|c|c|c|}
\hline \multirow[t]{4}{*}{ Characteristic/behavior } & \multirow{2}{*}{\multicolumn{4}{|c|}{$\begin{array}{l}\text { Mexico } \\
n=316\end{array}$}} & \multirow{4}{*}{$\begin{array}{l}\text { Crude } \\
\text { OR }\end{array}$} & \multirow{4}{*}{$\begin{array}{l}95 \% \text { confidence } \\
\text { interval }\end{array}$} & \multirow{2}{*}{\multicolumn{4}{|c|}{$\begin{array}{l}\text { California } \\
n=287\end{array}$}} & \multirow{4}{*}{$\begin{array}{l}\text { Crude } \\
\text { OR }\end{array}$} & \multirow{4}{*}{$\begin{array}{l}95 \% \text { confidence } \\
\text { interval }\end{array}$} \\
\hline & & & & & & & & & & & & \\
\hline & \multicolumn{2}{|c|}{$<85$ th $\%$} & \multicolumn{2}{|c|}{$\geq 85$ th $\%$} & & & \multicolumn{2}{|c|}{$<85$ th $\%$} & \multicolumn{2}{|c|}{$\geq 85$ th $\%$} & & \\
\hline & $n$ & $(\%)$ & $n$ & $(\%)$ & & & $n$ & $(\%)$ & $n$ & $(\%)$ & & \\
\hline Total $^{\mathrm{a}}$ & 269 & $(85.1)$ & 47 & $(14.9)$ & & & 134 & $(46.7)$ & 153 & $(53.3)$ & & \\
\hline \multicolumn{13}{|l|}{ Sex } \\
\hline Male & 121 & $(81.2)$ & 28 & $(18.8)$ & 1.0 & & 65 & $(48.2)$ & 70 & $(51.9)$ & 1.0 & \\
\hline Female & 148 & $(88.6)$ & 19 & (11.4) & 0.6 & $(0.3,1.0)^{\ddagger}$ & 69 & $(45.4)$ & 83 & $(54.6)$ & 1.1 & $(0.7,1.8)$ \\
\hline \multicolumn{13}{|l|}{ Mother's weight status } \\
\hline Normal & 74 & (94.9) & 4 & $(5.1)$ & 1.0 & & 22 & $(59.5)$ & 15 & $(40.5)$ & 1.0 & \\
\hline Overweight & 113 & $(85.6)$ & 19 & $(14.4)$ & 3.1 & $(1.0,9.5)^{\dagger}$ & 60 & $(54.6)$ & 50 & $(45.5)$ & 1.2 & $(0.6,2.6)$ \\
\hline Obese & 82 & $(77.4)$ & 24 & $(22.6)$ & 5.4 & $(1.8,16.3)^{\dagger}$ & 52 & $(37.1)$ & 88 & $(62.9)$ & 2.5 & $(1.2,5.2)^{\dagger}$ \\
\hline \multicolumn{13}{|l|}{ Exclusively breastfed $\geq 6$ months } \\
\hline No & 83 & $(83.0)$ & 17 & $(17.0)$ & 1.3 & $(0.7,2.4)$ & 17 & $(48.6)$ & 18 & $(51.4)$ & 0.9 & $(0.5,1.9)$ \\
\hline Yes & 186 & $(86.1)$ & 30 & $(13.9)$ & 1.0 & & 116 & $(46.4)$ & 134 & $(53.6)$ & 1.0 & \\
\hline \multicolumn{13}{|l|}{ Socioeconomic Status ${ }^{\mathrm{b}}$} \\
\hline Tertile 1 & 95 & $(90.5)$ & 10 & $(9.5)$ & 1.0 & & 62 & $(47.0)$ & 70 & $(53.0)$ & 1.0 & \\
\hline Tertile 2 & 87 & $(83.7)$ & 17 & $(16.4)$ & 1.9 & $(0.8,4.3)$ & 27 & $(45.0)$ & 33 & $(55.0)$ & 1.1 & $(0.6,2.0)$ \\
\hline Tertile 3 & 85 & $(81.0)$ & 20 & $(19.1)$ & 2.2 & $(1.0,5.0)^{\dagger}$ & 44 & $(46.8)$ & 50 & $(53.2)$ & 1.0 & $(0.6,1.7)$ \\
\hline \multicolumn{13}{|l|}{ Mother's education } \\
\hline Elementary or less & 187 & $(86.2)$ & 30 & $(13.8)$ & 1.0 & & 61 & $(43.0)$ & 81 & $(57.0)$ & 1.0 & \\
\hline Middle or high school & 76 & $(81.7)$ & 17 & $(18.3)$ & 1.4 & $(0.7,2.7)$ & 53 & $(52.5)$ & 48 & $(47.5)$ & 0.7 & $(0.4,1.1)$ \\
\hline High school graduate or more & 6 & $(100.0)$ & 0 & $(0.0)$ & & & 20 & $(45.5)$ & 24 & $(54.6)$ & 0.9 & $(0.5,1.8)$ \\
\hline \multicolumn{13}{|l|}{ Married or living as married } \\
\hline No & 20 & $(95.2)$ & 1 & $(4.8)$ & 0.3 & $(0.0,2.1)$ & 18 & $(56.3)$ & 14 & $(43.8)$ & 0.6 & $(0.3,1.4)$ \\
\hline Yes & 249 & $(84.4)$ & 46 & $(15.6)$ & 1.0 & & 116 & $(45.5)$ & 139 & $(54.5)$ & 1.0 & \\
\hline \multicolumn{13}{|l|}{ Mother's work status } \\
\hline Not working & 175 & $(86.6)$ & 27 & $(13.4)$ & 1.0 & & 39 & $(46.4)$ & 45 & $(53.6)$ & 1.0 & \\
\hline Work less than $20 \mathrm{~h} /$ week & 42 & $(77.8)$ & 12 & $(22.2)$ & 1.9 & $(0.9,4.0)$ & 10 & $(66.7)$ & 5 & $(33.3)$ & 0.4 & $(0.1,1.4)$ \\
\hline Work $20-40 \mathrm{~h} /$ week & 20 & $(90.9)$ & 2 & $(9.1)$ & 0.6 & $(0.1,2.9)$ & 30 & $(44.8)$ & 37 & $(55.2)$ & 1.1 & $(0.6,2.0)$ \\
\hline Work more than $40 \mathrm{~h} /$ week & 32 & $(84.2)$ & 6 & $(15.8)$ & 1.2 & $(0.5,3.2)$ & 55 & $(45.5)$ & 66 & $(54.6)$ & 1.0 & $(0.6,1.8)$ \\
\hline \multicolumn{13}{|l|}{ Household food insecurity } \\
\hline Secure & 71 & $(91.0)$ & 7 & $(9.0)$ & 1.0 & & 81 & $(47.1)$ & 91 & $(52.9)$ & 1.0 & \\
\hline Low food security & 127 & $(85.2)$ & 22 & $(14.8)$ & 1.8 & $(0.7,4.3)$ & 42 & $(48.3)$ & 45 & $(51.7)$ & 1.0 & $(0.6,1.6)$ \\
\hline Very low food security & 71 & $(79.8)$ & 18 & $(20.2)$ & 2.6 & $(1.0,6.5)^{\dagger}$ & 11 & $(39.3)$ & 17 & $(60.7)$ & 1.4 & $(0.6,3.1)$ \\
\hline Daily TV time & & & & & & & & & & & & \\
\hline $1 \mathrm{~h}$ or less & 120 & $(87.0)$ & 18 & $(13.0)$ & 1.0 & & 27 & $(45.9)$ & 36 & $(54.1)$ & 1.0 & \\
\hline $1-2 \mathrm{~h}$ & 68 & $(80.0)$ & 17 & $(20.0)$ & 1.7 & $(0.8,3.4)$ & 42 & $(49.5)$ & 45 & $(50.5)$ & 0.9 & $(0.5,1.5)$ \\
\hline Greater than $2 \mathrm{~h}$ & 81 & $(87.1)$ & 12 & $(12.9)$ & 1.0 & $(0.5,2.2)$ & 65 & $(44.6)$ & 72 & $(55.5)$ & 1.1 & $(0.6,1.9)$ \\
\hline Time spent playing outside & & & & & & & & & & & & \\
\hline $1 \mathrm{~h}$ or less & 31 & $(75.6)$ & 10 & $(24.4)$ & 1.0 & & 50 & $(44.3)$ & 63 & $(55.8)$ & 1.0 & \\
\hline $2-3 \mathrm{~h}$ & 124 & $(87.9)$ & 17 & $(12.1)$ & 0.4 & $(0.2,1.0)$ & 63 & $(50.8)$ & 61 & $(49.2)$ & 0.8 & $(0.5,1.3)$ \\
\hline $4 \mathrm{~h}$ or more & 114 & $(85.1)$ & 20 & (14.9) & 0.5 & $(0.2,1.3)$ & 21 & $(42.0)$ & 29 & $(58.0)$ & 1.1 & $(0.6,2.1)$ \\
\hline Soda consumption ${ }^{\mathrm{c}}$ & & & & & & & & & & & & \\
\hline Less than 1 per week & 68 & $(93.2)$ & 5 & $(6.9)$ & 1.0 & & 55 & $(50.0)$ & 55 & $(50.0)$ & 1.0 & \\
\hline $1-6$ per week & 153 & $(81.0)$ & 36 & $(19.1)$ & 3.2 & $(1.2,8.5)^{\dagger}$ & 70 & $(45.8)$ & 83 & $(54.3)$ & 1.2 & $(0.7,1.9)$ \\
\hline 1 a day or more & 48 & $(88.9)$ & 6 & $(11.1)$ & 1.7 & $(0.5,5.9)$ & 9 & $(37.5)$ & 15 & $(62.5)$ & 1.7 & $(0.7,4.1)$ \\
\hline
\end{tabular}


Table 2 continued

\begin{tabular}{|c|c|c|c|c|c|c|c|c|c|c|c|c|}
\hline \multirow[t]{4}{*}{ Characteristic/behavior } & \multirow{2}{*}{\multicolumn{4}{|c|}{$\begin{array}{l}\text { Mexico } \\
n=316\end{array}$}} & \multirow{4}{*}{$\begin{array}{l}\text { Crude } \\
\text { OR }\end{array}$} & \multirow{4}{*}{$\begin{array}{l}95 \% \text { confidence } \\
\text { interval }\end{array}$} & \multirow{2}{*}{\multicolumn{4}{|c|}{$\begin{array}{l}\text { California } \\
n=287\end{array}$}} & \multirow{4}{*}{$\begin{array}{l}\text { Crude } \\
\text { OR }\end{array}$} & \multirow{4}{*}{$\begin{array}{l}95 \% \text { confidence } \\
\text { interval }\end{array}$} \\
\hline & & & & & & & & & & & & \\
\hline & \multicolumn{2}{|c|}{$<85$ th $\%$} & \multicolumn{2}{|c|}{$\geq 85 \mathrm{th} \%$} & & & \multicolumn{2}{|c|}{$<85$ th $\%$} & \multicolumn{2}{|c|}{$\geq 85$ th $\%$} & & \\
\hline & $n$ & $(\%)$ & $n$ & $(\%)$ & & & $n$ & $(\%)$ & $n$ & $(\%)$ & & \\
\hline \multicolumn{13}{|c|}{ Other sweetened beverage consumption ${ }^{c}$} \\
\hline Less than 1 per day & 166 & $(84.7)$ & 30 & $(15.3)$ & 1.0 & & 50 & $(44.6)$ & 62 & $(55.4)$ & 1.0 & \\
\hline 1 to less than 2 per day & 61 & $(85.9)$ & 10 & $(14.1)$ & 0.9 & $(0.4,2.0)$ & 23 & $(42.6)$ & 31 & $(57.4)$ & 1.1 & $(0.6,2.1)$ \\
\hline 2 or more perday & 42 & $(85.7)$ & 7 & $(14.3)$ & 0.9 & $(0.4,2.2)$ & 61 & $(50.4)$ & 60 & $(49.6)$ & 0.8 & $(0.5,1.3)$ \\
\hline \multicolumn{13}{|l|}{ Fast food consumption } \\
\hline Less than once per week & 260 & $(86.4)$ & 46 & $(13.7)$ & 1.0 & & 47 & $(45.2)$ & 57 & $(54.8)$ & 1.0 & \\
\hline Once a week or more & 8 & $(77.3)$ & 1 & $(22.7)$ & 1.9 & $(0.8,4.1)$ & 86 & $(47.5)$ & 95 & $(52.5)$ & 0.9 & $(0.6,1.5)$ \\
\hline \multicolumn{13}{|c|}{ Sweets and snacks consumption ${ }^{c}$} \\
\hline Less than 1 per day & 47 & $(81.0)$ & 11 & $(19.0)$ & 1.0 & & 18 & $(43.9)$ & 23 & $(56.1)$ & 1.0 & \\
\hline 1 to less than 2 per day & 83 & $(89.3)$ & 10 & $(10.8)$ & 0.5 & $(0.2,1.3)$ & 36 & $(44.4)$ & 45 & $(55.6)$ & 1.0 & $(0.5,1.2)$ \\
\hline 2 or more perday & 139 & $(84.2)$ & 26 & $(15.8)$ & 0.8 & $(0.4,1.7)$ & 80 & $(48.5)$ & 85 & $(51.5)$ & 0.8 & $(0.4,1.7)$ \\
\hline \multicolumn{13}{|l|}{ Fruit consumption $^{c}$} \\
\hline Less than 1 per day & 96 & $(85.0)$ & 17 & $(15.0)$ & 1.0 & & 9 & $(56.3)$ & 7 & $(43.8)$ & 1.0 & \\
\hline $1-2$ per day & 96 & $(86.5)$ & 19 & $(16.5)$ & 1.1 & $(0.5,2.3)$ & 21 & $(48.8)$ & 22 & $(51.2)$ & 1.3 & $(0.4,4.3)$ \\
\hline 2 per day or more & 77 & $(87.5)$ & 11 & $(12.5)$ & 0.8 & $(0.4,1.8)$ & 104 & $(45.6)$ & 124 & $(54.4)$ & 1.5 & $(0.6,4.3)$ \\
\hline \multicolumn{13}{|l|}{ Vegetable consumption $^{c}$} \\
\hline Less than 1 per day & 21 & $(84.0)$ & 4 & $(16.0)$ & 1.0 & & 18 & $(52.9)$ & 16 & $(47.1)$ & 1.0 & \\
\hline $1-2$ per day & 68 & $(82.9)$ & 14 & $(17.1)$ & 1.1 & $(0.3,3.6)$ & 27 & $(39.7)$ & 41 & $(60.3)$ & 1.7 & $(0.7,3.9)$ \\
\hline 2 per day or more & 180 & $(86.1)$ & 29 & (13.9) & 0.8 & $(0.3,2.6)$ & 89 & $(48.1)$ & 96 & $(51.9)$ & 1.2 & $(0.6,2.5)$ \\
\hline \multicolumn{13}{|l|}{ Mother's years in the US } \\
\hline $5-10$ years & & & & & & & 73 & $(50.3)$ & 72 & $(49.7)$ & 1.0 & \\
\hline $11-15$ years & & & & & & & 35 & $(39.3)$ & 54 & $(60.7)$ & 1.6 & $(0.9,2.7)^{*}$ \\
\hline $16+$ years & & & & & & & 26 & $(49.1)$ & 27 & $(50.9)$ & 1.1 & $(0.6,2.0)$ \\
\hline \multicolumn{13}{|c|}{ Family member currently in $\mathrm{US}^{\mathrm{d}}$} \\
\hline No & 116 & $(82.9)$ & 24 & $(17.1)$ & 1.0 & & & & & & & \\
\hline Yes & 153 & (86.9) & 23 & (13.1) & 0.7 & $(0.4,1.4)$ & & & & & & \\
\hline
\end{tabular}

${ }^{\text {a }}$ Row percentages

b Socioeconomic status was determined by a principal component analysis of housing characteristics and assets in Proyecto Mariposa

c Mother's report of how many times the child consumed the item, serving sizes were not specified

${ }^{\mathrm{d}}$ Family member includes child's father or the current head of household and the mother's grandparents, parents, siblings, or other children

${ }^{\dagger} P<0.05 ;{ }^{\star} P<0.10$

odds of being overweight or obese compared to children in the lowest SES tertile (95\% CI: 1.3, 7.5); and children experiencing very low food security had almost four times the odds compared to children from food secure households (OR 3.8 95\% CI: 1.4, 10.6).

\section{Discussion}

The main findings of this binational study were: (1) the prevalence of childhood obesity was much higher among children of Mexican descent in the US than in Mexico; (2) maternal obesity was a determinant of childhood obesity in both settings and (3) in Mexico, male gender, high SES and low food security were determinants of childhood obesity.

The difference in prevalence of overweight and obesity was much greater than expected based on previous research. One-third of Mexican-American children ages two to five were above the 85th percentile in NHANES (2003-2004) [6] compared to 53\% of 5-year-olds in California. Also, the 2003 Mexican National Social Welfare Survey, which surveys Oportunidades participants, found that $24 \%$ of non-indigenous children $49-60$ months old were classified as overweight or obese, using International 
Table 3 Multivariate model of predictors of overweight and obesity ( $\geq 85$ th percentile), California (Salinas, CA) and Mexico (Guanajuato, Jalisco, and Michoacán, Mexico) 2006

\begin{tabular}{|c|c|c|c|c|}
\hline & \multicolumn{2}{|l|}{ Mexico } & \multicolumn{2}{|l|}{ California } \\
\hline & Adjusted odds ratio ${ }^{\mathrm{a}}$ & $(95 \% \mathrm{CI})$ & Adjusted odds ratio ${ }^{a}$ & $(95 \% \mathrm{CI})$ \\
\hline \multicolumn{5}{|l|}{ Mother's weight status } \\
\hline Normal & ref & & ref & \\
\hline Overweight & 3.1 & $(1.0,9.6)$ & 1.2 & $(0.6,2.6)$ \\
\hline Obese & 5.5 & $(1.8,17.1)^{*}$ & 2.4 & $(1.1,5.3)^{\dagger}$ \\
\hline \multicolumn{5}{|l|}{ Sex } \\
\hline Male & ref & & ref & \\
\hline Female & 0.5 & $(0.2,0.9)^{\dagger}$ & 1.1 & $(0.7,1.8)$ \\
\hline \multicolumn{5}{|l|}{ Socioeconomic status } \\
\hline Tertile 1 & ref & & ref & \\
\hline Tertile 2 & 2.0 & $(0.8,4.9)$ & 1.1 & $(0.6,2.1)$ \\
\hline Tertile 3 & 3.1 & $(1.3,7.5)^{*}$ & 1.0 & $(0.5,1.7)$ \\
\hline \multicolumn{5}{|l|}{ Household food insecurity } \\
\hline Secure & ref & & ref & \\
\hline Low food security & 2.1 & $(0.8,5.2)$ & 0.8 & $(0.5,1.4)$ \\
\hline Very low food security & 3.8 & $(1.4,10.6)^{\ddagger}$ & 1.3 & $(0.5,3.0)$ \\
\hline
\end{tabular}

Obesity Task Force (IOTF) criteria [55], which is higher than the $15 \%$ we observed. A prevalence of $15 \%$ is what is normally expected to be at or above the 85th percentile. Thus, this population may not be representative of other non-indigenous areas where the prevalence is higher. Similarly, 5\% of non-indigenous children 49-60 months old were concurrently obese and stunted in this national survey, which occurred in less than $1 \%$ of the children in our Mexican sample (data not shown).

In Mexico, male children were more likely to be overweight compared to female children. Nationally representative surveys in Mexico as well as Mexican-Americans in the US have also documented this gender difference in childhood obesity. The 2003 Mexican National Social Welfare Survey showed that males were more likely to be obese than females [55]. Similarly, among MexicanAmerican children ages two to five surveyed in NHANES (2003-2004), 38\% of males were overweight or obese compared to $27 \%$ of females [6]. However, we did not document a sex difference among children in the California sample. It is possible that in the face of limited food resources in Mexico, resources are more likely to be devoted to boys than girls but this may not be the case in California.

Higher SES was associated with increased odds of childhood obesity in the Mexican sample. Two other studies of Oportunidades participants have also found that
SES was positively associated with child obesity $[55,56]$. Families with relatively higher SES levels may have increased resources to procure foods that may lead to obesity. In fact, further analyses of these data showed that children in the highest SES tertile in Mexico consumed sweets and fried snacks significantly more often than children in the lower SES tertiles $(P=0.03)$. There was no difference in the odds of overweight or obesity according to SES among children in the California sample. Although lower SES has been associated with an increased risk of childhood obesity in the US [57], it may not apply within a predominantly poor sample, such as this one, in contrast to Mexico.

Additionally, in Mexico, where approximately threequarters of the households experienced some degree of food insecurity in the last 12 months, we found that food insecurity was associated with child obesity. Food insecurity could lead to childhood obesity through mechanisms that have been proposed in the literature, such as over reliance on inexpensive, energy-dense foods [58], overeating when food is available [58] and changes in metabolism that favor more efficient use of energy [59]. A previous analysis using these data revealed that children experiencing low or very low food security consumed less of the majority of food categories except for beans compared to children from food secure households [60]. Thus, explanations for the food insecurity-obesity connection 
appear to be explained by hypotheses involving cyclical eating patterns or metabolic changes, which would not be captured by a food frequency questionnaire.

Only one study has been conducted on household food insecurity and weight status among children in Mexico. This study found that household food insecurity was associated with being overweight among children aged 9-15 in a low-income area of Mexico City [61]. However, research on food insecurity and child weight status in the US and other countries has been equivocal, with some studies showing that food insecurity is associated with obesity or a higher BMI [59, 62-66], other studies showing an association between food insecurity and lower BMI [67, 68], and others showing no association [69-72]. Different findings may be related to differences in study populations; the participants in our study were all participants in the Oportunidades program and from the lowest 20th percentile of income in Mexico. Household food insecurity was not associated with child weight status in the California sample, although further analyses showed that it was associated with maternal obesity (data not shown). It could be that food insecurity was not as severe in the California sample and thus children were spared the effects. Longitudinal and in-depth qualitative research is needed to better understand the association between food security and child weight status.

We found that high SES and household food insecurity were both independently associated with childhood obesity in Mexico. This may be a result of the characteristics of the Mexican sample. All families in the Mexico sample were poor as they were drawn from the lowest 20th percentile of income in Mexico and a high percentage of mothers in each SES tertile reported food insecurity (83-64\%). Although our numbers were small, it appeared that the prevalence of overweight and obesity for those in food insecure households was similar among all levels of SES (19-22\%) i.e. there was no evidence of interaction between food insecurity and SES. Alternatively, this may be explained by the measure of food insecurity. Because food insecurity measures mothers' perception of having enough food for the family, it is possible that mothers in the highest SES group still worry about not having enough food. Additionally, it could be that previously mentioned mechanisms, such as cyclical eating and metabolic changes, operate at all levels of SES. Additional research exploring the economic factors explaining the associations of SES and food insecurity with obesity are needed.

Maternal weight status was a strong correlate of child weight status in both the Mexican-American and Mexican children sampled, which has also been shown in other studies [73, 74]. Although this may suggest a genetic component to weight status, mother's weight status may also reflect characteristics of the environment shared with the child, which may be more important [75]. In addition, maternal weight status may also influence child weight status during the prenatal period [76]. Research has shown that overweight and obese mothers are more likely to gain excessive weight during pregnancy and excessive weight gain during pregnancy has been shown to be associated with subsequent childhood overweight [76]. Research is needed to examine the effect of gestational weight gain in Mexican mothers.

We did not find evidence to support the acculturation hypothesis in this study using maternal length of residence in the US as a proxy. Other research in younger children has also failed to document an association [20, 21, 23, 26]. It is possible that we did not observe an association between acculturation and children's weight status because the California sample was recruited from a relatively homogenous population of low-income Mexican immigrant farmworkers. Alternatively, time in the US may have been an insufficient indicator for measuring the domain(s) of acculturation important for childhood obesity [77].

This study had several limitations. First, although we made every attempt to recruit comparable samples and collect equivalent data in each site, our samples may have been too different and data collection instruments incapable of collecting the same information in both sites. Thus, making comparisons may be difficult. Second, we relied on maternal report of diet and physical activity. Mothers may not have been able to accurately report their child's regular dietary intake or physical activity levels especially if she worked outside the home, as was the case for $58 \%$ of the mothers in California and $34 \%$ of the mothers in Mexico. Furthermore, although diet was measured using previously validated instruments, the physical activity variable was not. Third, we used the short form to measure household food insecurity, which may not have provided as complete of a picture of food insecurity compared to the long form. Finally, this was a cross-sectional study, which does not permit us to infer any causality.

Our binational study findings clearly show that children of Mexican descent living in California are at increased risk for being overweight compared to children that remain in sending communities in Mexico. Our results suggest that successful programs and policies addressing childhood obesity in both countries should include mothers or perhaps the entire family. Additionally, interventions on both sides of the border should take into consideration the effects of gender, SES and food security on obesity among children in Mexico. For example, in the US, interventions should acknowledge that, obesity, although undesirable from a health perspective, may be a sign of desirable increases in SES in immigrants' communities of origin. 
Acknowledgments This research was made possible by funding provided by National Institute of Environmental Health Sciences (PO1 ES009605), the Environmental Protection Agency (RD 83171001), UC-Mexus, University of California Berkeley Health Initiative of the Americas, University of California Berkeley Center for Latino Policy Studies, University of California Institute for the Study of Social Change, and the W.K. Kellogg Foundation Postdoctoral Fellowship.

Open Access This article is distributed under the terms of the Creative Commons Attribution Noncommercial License which permits any noncommercial use, distribution, and reproduction in any medium, provided the original author(s) and source are credited.

\section{References}

1. Flegal KM, Carroll MD, Ogden CL, Johnson CL. Prevalence and trends in obesity among US adults, 1999-2000. JAMA. 2002; 288:1723-7.

2. Ogden CL, Flegal KM, Carroll MD, Johnson CL. Prevalence and trends in overweight among US children and adolescents, 19992000. JAMA. 2002;288:1728-32.

3. Hedley AA, Ogden CL, Johnson CL, Carroll MD, Curtin LR, Flegal KM. Prevalence of overweight and obesity among US children, adolescents, and adults, 1999-2002. JAMA. 2004;291: 2847-50.

4. Strauss RS, Pollack HA. Epidemic increase in childhood overweight, 1986-1998. JAMA. 2001;286:2845-8.

5. Flegal KM, Ogden CL, Carroll MD. Prevalence and trends in overweight in Mexican-American adults and children. Nutr Rev. 2004;62:S144-8.

6. Ogden CL, Carroll MD, Curtin LR, McDowell MA, Tabak CJ, Flegal KM. Prevalence of overweight and obesity in the United States, 1999-2004. JAMA. 2006;295:1549-55.

7. Guo SS, Wu W, Chumlea WC, Roche AF. Predicting overweight and obesity in adulthood from body mass index values in childhood and adolescence. Am J Clin Nutr. 2002;76:653-8.

8. Deshmukh-Taskar P, Nicklas TA, Morales M, Yang SJ, Zakeri I, Berenson GS. Tracking of overweight status from childhood to young adulthood: the Bogalusa Heart Study. Eur J Clin Nutr. 2006;60:48-57.

9. Nader PR, O’Brien M, Houts R, Bradley R, Belsky J, Crosnoe R, et al. Identifying risk for obesity in early childhood. Pediatrics. 2006;118:e594-601.

10. Sundquist J, Winkleby MA. Cardiovascular risk factors in Mexican American adults: a transcultural analysis of NHANES III, 1988-1994. Am J Public Health. 1999;89:723-30.

11. Davidson JA, Kannel WB, Lopez-Candales A, Morales L, Moreno PR, Ovalle F, et al. Avoiding the looming latino/hispanic cardiovascular health crisis: a call to action. J Cardiometab Syndr. 2007;2:238-43.

12. Hunt KJ, Resendez RG, Williams K, Haffner SM, Stern MP, Hazuda HP. All-cause and cardiovascular mortality among Mexican-American and non-hispanic white older participants in the San Antonio Heart Study-evidence against the "hispanic paradox". Am J Epidemiol. 2003;158:1048-57.

13. Hunt KJ, Williams K, Resendez RG, Hazuda HP, Haffner SM, Stern MP. All-cause and cardiovascular mortality among diabetic participants in the San Antonio Heart Study: evidence against the "hispanic paradox". Diabetes Care. 2002;25:1557-63.

14. Winkleby MA, Fortmann SP, Rockhill B. Health-related risk factors in a sample of Hispanics and whites matched on sociodemographic characteristics. The Stanford five-city project. Am J Epidemiol. 1993;137:1365-75.
15. Popkin BM, Udry JR. Adolescent obesity increases significantly in second and third generation U.S. immigrants: the National Longitudinal Study of Adolescent Health. J Nutr. 1998;128: 701-6.

16. Kaiser LL, Melgar-Quinonez HR, Lamp CL, Johns MC, Harwood JO. Acculturation of Mexican-American mothers influences child feeding strategies. J Am Diet Assoc. 2001;101:542-7.

17. Haas JS, Lee LB, Kaplan CP, Sonneborn D, Phillips KA, Liang SY. The association of race, socioeconomic status, and health insurance status with the prevalence of overweight among children and adolescents. Am J Public Health. 2003;93:2105-10.

18. Gordon-Larsen P, Harris KM, Ward DS, Popkin BM. Acculturation and overweight-related behaviors among Hispanic immigrants to the US: the National Longitudinal Study of Adolescent Health. Soc Sci Med. 2003;57:2023-34.

19. Nelson JA, Chiasson MA, Ford V. Childhood overweight in a New York City WIC population. Am J Public Health. 2004;94: 458-62.

20. Ariza AJ, Chen EH, Binns HJ, Christoffel KK. Risk factors for overweight in five- to six-year-old Hispanic-American children: a pilot study. J Urban Health. 2004;81:150-61.

21. Warner ML, Harley K, Bradman A, Vargas G, Eskenazi B. Soda consumption and overweight status of 2-year-old MexicanAmerican children in California. Obesity (Silver Spring). 2006;14: 1966-74.

22. Van Hook J, Balistreri KS. Immigrant generation, socioeconomic status, and economic development of countries of origin: a longitudinal study of body mass index among children. Soc Sci Med. 2007;65:976-89.

23. Duerksen SC, Elder JP, Arredondo EM, Ayala GX, Slymen DJ, Campbell NR, et al. Family restaurant choices are associated with child and adult overweight status in Mexican-American families. J Am Diet Assoc. 2007;107:849-53.

24. Hernandez-Valero MA, Wilkinson AV, Forman MR, Etzel CJ, Cao Y, Barcenas CH, et al. Maternal BMI and country of birth as indicators of childhood obesity in children of Mexican origin. Obesity (Silver Spring). 2007;15:2512-9.

25. Butte NF, Cai G, Cole SA, Wilson TA, Fisher JO, Zakeri IF, et al. Metabolic and behavioral predictors of weight gain in Hispanic children: the Viva la Familia Study. Am J Clin Nutr. 2007;85: $1478-85$.

26. Kimbro RT, Brooks-Gunn J, McLanahan S. Racial and ethnic differentials in overweight and obesity among 3-year-old children. Am J Public Health. 2007;97:298-305.

27. Fuentes-Afflick E, Hessol NA. Overweight in young Latino children. Arch Med Res. 2008;39:511-8.

28. Abraido-Lanza AF, Chao MT, Florez KR. Do healthy behaviors decline with greater acculturation? Implications for the Latino mortality paradox. Soc Sci Med. 2005;61:1243-55.

29. Dixon LB, Sundquist J, Winkleby M. Differences in energy, nutrient, and food intakes in a US sample of Mexican-American women and men: findings from the Third National Health and Nutrition Examination Survey, 1988-1994. Am J Epidemiol. 2000;152:548-57.

30. Guendelman S, Abrams B. Dietary intake among MexicanAmerican women: generational differences and a comparison with white non-Hispanic women. Am J Public Health. 1995;85:20-5.

31. Neuhouser ML, Thompson B, Coronado GD, Solomon CC. Higher fat intake and lower fruit and vegetables intakes are associated with greater acculturation among Mexicans living in Washington State. J Am Diet Assoc. 2004;104:51-7.

32. Sundquist J, Winkleby M. Country of birth, acculturation status and abdominal obesity in a national sample of Mexican-American women and men. Int J Epidemiol. 2000;29:470-7.

33. Barcenas $\mathrm{CH}$, Wilkinson AV, Strom SS, Cao Y, Saunders KC, Mahabir S, et al. Birthplace, years of residence in the United 
States, and obesity among Mexican-American adults. Obesity (Silver Spring). 2007;15:1043-52.

34. Fuentes-Afflick E. Obesity among Latino preschoolers: do children outgrow the "epidemiologic paradox"? Arch Pediatr Adolesc Med. 2006;160:656-7.

35. Popkin BM, Gordon-Larsen P. The nutrition transition: worldwide obesity dynamics and their determinants. Int J Obes Relat Metab Disord. 2004;28(Suppl 3):S2-9.

36. Institute of Medicine. Joint U.S.-Mexico workshop on preventing obesity in children and youth of mexican origin: summary. Washington DC: The National Academies Press; 2007.

37. Filozof C, Gonzalez C, Sereday M, Mazza C, Braguinsky J. Obesity prevalence and trends in Latin-American countries. Obes Rev. 2001;2:99-106.

38. Rivera JA, Barquera S, Campirano F, Campos I, Safdie M, Tovar V. Epidemiological and nutritional transition in Mexico: rapid increase of non-communicable chronic diseases and obesity. Public Health Nutr. 2002;5:113-22.

39. Olaiz G, Rivera JA, Shamah T, Rojas R, Villalpando S, Hernandez M, Sepulveda J. Encuesta Nacional de Salud y Nutricion, 2006. Cuernavaca, Mexico: Instituto Nacional de Salud Publica; 2006.

40. Jimenez-Cruz A, Bacardi-Gascon M, Spindler AA. Obesity and hunger among Mexican-Indian migrant children on the USMexico border. Int J Obes Relat Metab Disord. 2003;27:740-7.

41. Ayala GX, Elder JP, Campbell NR, Slymen DJ, Roy N, Engelberg $\mathrm{M}$, et al. Correlates of body mass index and waist-to-hip ratio among Mexican women in the United States: implications for intervention development. Womens Health Issues. 2004;14: 155-64.

42. Perichart-Perera O, Balas-Nakash M, Schiffman-Selechnik E, Barbato-Dosal A, Vadillo-Ortega F. Obesity increases metabolic syndrome risk factors in school-aged children from an urban school in Mexico city. J Am Diet Assoc. 2007;107:81-91.

43. Eskenazi B, Gladstone EA, Berkowitz GS, Drew CH, Faustman EM, Holland NT, et al. Methodologic and logistic issues in conducting longitudinal birth cohort studies: lessons learned from the Centers for Children's Environmental Health and Disease Prevention Research. Environ Health Perspect. 2005;113:1419-29.

44. Eskenazi B, Harley K, Bradman A, Weltzien E, Jewell NP, Barr $\mathrm{DB}$, et al. Association of in utero organophosphate pesticide exposure and fetal growth and length of gestation in an agricultural population. Environ Health Perspect. 2004;112:1116-24.

45. INEGI. Conteo de poblacion y vivienda 2005 [Population and Housing Census 2005]. 2005; http://www.inegi.gob.mx/inegi/ default.aspx?s=est\&c=10202. Accessed July 1, 2008; 2008.

46. National Center for Health Statistics. CDC growth charts, United States; 2005.

47. Johnson-Taylor WL, Everhart JE. Modifiable environmental and behavioral determinants of overweight among children and adolescents: report of a workshop. Obesity (Silver Spring). 2006;14: 929-66.

48. WHO. Physical status: the use and interpretation of anthropometry. Geneva: World Health Organization; 1995.

49. Arcia E, Skinner M, Bailey D, Correa V. Models of acculturation and health behaviors among Latino immigrants to the US. Soc Sci Med. 2001;53:41-53.

50. Bickel G, Nord M, Price C, Hamilton W, Cook J. Guide to measuring household food security: United States Department of Agriculture Food and Nutrition Service, Office of Analysis, Nutrition, and Evaluation; 2000.

51. Harrison GG, Stormer A, Herman DR, Winham DM. Development of a spanish-language version of the U.S. household food security survey module. J Nutr. 2003;133:1192-7.

52. Falkingham J, Namazie C. Measuring health and poverty: a review of approaches to identifying the poor. London:
Department for International Development Health Systems Resource Centre; 2002.

53. Vyas S, Kumaranayake L. Constructing socio-economic status indices: how to use principal components analysis. Health Policy Plan. 2006;21:459-68.

54. Stata Statistical Software: Release 8.0 [computer program]. College Station, TX: Stata Corporation; 2003.

55. Fernald LC, Neufeld LM. Overweight with concurrent stunting in very young children from rural Mexico: prevalence and associated factors. Eur J Clin Nutr. 2007;61:623-32.

56. Fernald LC. Socio-economic status and body mass index in lowincome Mexican adults. Soc Sci Med. 2007;64:2030-42.

57. Singh GK, Kogan MD, Van Dyck PC, Siahpush M. Racial/ethnic, socioeconomic, and behavioral determinants of childhood and adolescent obesity in the United States: analyzing independent and joint associations. Ann Epidemiol. 2008;18:682-95.

58. Dinour LM, Bergen D, Yeh MC. The food insecurity-obesity paradox: a review of the literature and the role food stamps may play. J Am Diet Assoc. 2007;107:1952-61.

59. Alaimo K, Olson CM, Frongillo EA Jr. Low family income and food insufficiency in relation to overweight in US children: is there a paradox? Arch Pediatr Adolesc Med. 2001;155:1161-7.

60. Rosas LG, Harley K, Fernald LC, Guendelman S, Mejia F, Neufeld LM, et al. Dietary associations of household food insecurity among children of Mexican descent: results of a binational study. J Am Diet Assoc. 2009;109:2001-9.

61. Ortiz-Hernandez L, Acosta-Gutierrez MN, Nunez-Perez AE, Peralta-Fonseca N, Ruiz-Gomez Y. Food insecurity and obesity are positively associated in Mexico City school children. Rev Invest Clin. 2007;59:32-41.

62. Casey PH, Simpson PM, Gossett JM, Bogle ML, Champagne $\mathrm{CM}$, Connell $\mathrm{C}$, et al. The association of child and household food insecurity with childhood overweight status. Pediatrics. 2006;118:e1406-13.

63. Dietz WH. Does hunger cause obesity? Pediatrics. 1995;95: 766-7.

64. Smith C, Richards R. Dietary intake, overweight status, and perceptions of food insecurity among homeless Minnesotan youth. Am J Hum Biol. 2008;20:550-63.

65. Jyoti DF, Frongillo EA, Jones SJ. Food insecurity affects school children's academic performance, weight gain, and social skills. J Nutr. 2005;135:2831-9.

66. Bronte-Tinkew J, Zaslow M, Capps R, Horowitz A, McNamara M. Food insecurity works through depression, parenting, and infant feeding to influence overweight and health in toddlers. J Nutr. 2007;137:2160-5.

67. Matheson DM, Varady J, Varady A, Killen JD. Household food security and nutritional status of Hispanic children in the fifth grade. Am J Clin Nutr. 2002;76:210-7.

68. Isanaka S, Mora-Plazas M, Lopez-Arana S, Baylin A, Villamor E. Food insecurity is highly prevalent and predicts underweight but not overweight in adults and school children from Bogota, Colombia. J Nutr. 2007;137:2747-55.

69. Whitaker RC, Orzol SM. Obesity among US urban preschool children: relationships to race, ethnicity, and socioeconomic status. Arch Pediatr Adolesc Med. 2006;160:578-84.

70. Martin KS, Ferris AM. Food insecurity and gender are risk factors for obesity. J Nutr Educ Behav. 2007;39:31-6.

71. Gundersen C, Lohman BJ, Eisenmann JC, Garasky S, Stewart SD. Child-specific food insecurity and overweight are not associated in a sample of 10- to 15-year-old low-income youth. J Nutr. 2008;138:371-8.

72. Gundersen C, Lohman BJ, Garasky S, Stewart S, Eisenmann J. Food security, maternal stressors, and overweight among lowincome US children: results from the National Health and 
Nutrition Examination Survey (1999-2002). Pediatrics. 2008; 122:e529-40.

73. Danielzik S, Czerwinski-Mast M, Langnase K, Dilba B, Muller MJ. Parental overweight, socioeconomic status and high birth weight are the major determinants of overweight and obesity in 5-7 y-old children: baseline data of the Kiel Obesity Prevention Study (KOPS). Int J Obes Relat Metab Disord. 2004;28:1494-502.

74. Strauss RS, Knight J. Influence of the home environment on the development of obesity in children. Pediatrics. 1999;103:e85.
75. Franks PW, Ravussin E, Hanson RL, Harper IT, Allison DB, Knowler WC, et al. Habitual physical activity in children: the role of genes and the environment. Am J Clin Nutr. 2005;82:901-8.

76. Oken E, Taveras EM, Kleinman KP, Rich-Edwards JW, Gillman MW. Gestational weight gain and child adiposity at age 3 years. Am J Obstet Gynecol. 2007;196(322):e321-8.

77. Abraido-Lanza AF, Armbrister AN, Florez KR, Aguirre AN. Toward a theory-driven model of acculturation in public health research. Am J Public Health. 2006;96:1342-6. 